changes in conformation, or the $60-70 \mathrm{~S}$ RNA comprises an assembly of $30-35 \mathrm{~S}$ subunits held together by heat labile, DMSO labile bonds-in short, hydrogen bonds. If the second model is correct, as is widely believed, and if relationships between sedimentation coefficients and molecular weight derived for small RNA molecules are valid it is simple to calculate that the 60-70S RNA has a molecular of weight about $10-12 \times 10^{6}$ and that the putative $30-35$ S RNA subunits have a molecular weight of about $3.3-3.5 \times 10^{6}$.

Two lines of evidence support the notion that the $60-70$ S RNA of RSV is an aggregate of subunits-in other words that the RSV genome is segmented; first, when chick fibroblasts are infected by two different strains of RSV recombinant virus particles form a considerable proportion of the progeny. This high frequency of recombination, which is also a characteristic of influenza virus - a virus with a single segmented RNA genome-can readily be explained if the RSV genome comprises three or four RNA chains, for in mixed infections progeny virions may assemble by taking combinations of RNA subunits of the two parental types. Second, Cheung et al. (1972) and Canaani et al. (1973) have just reported quite different physicochemical evidence which suggests that 30-35S RNA may be a precursor of 60-70S RNA.

Cheung et al. (Virology, 58, $51 ; 1972$ ) harvested Prague strain RSV from chick fibroblasts at $5,10,20,60$ and $180 \mathrm{~min}$ and $12 \mathrm{~h}$ and $24 \mathrm{~h}$ intervals. The $5 \mathrm{~min}$ harvest virus proved to contain little 68S RNA, but it contained a heterogenous RNA with a median sedimentation coefficient of 55-60S which on denaturation yielded $36 \mathrm{~S}$ RNA and RNA sedimenting between $36 \mathrm{~S}$ and $4 \mathrm{~S}$. By contrast $24 \mathrm{~h}$ harvest virus particles yielded a homogenous 68S RNA which denatured to yield homogenous $36 \mathrm{~S}$ and $4 \mathrm{~S}$ RNAs. Particles harvested at intermediate times yielded RNA patterns which were intermediate between these two extremes.

Canaani et al. (Proc. US Nat. Acad. Sci., 70, 401; 1973), who found themselves down wind, so to speak, of Cheung and his colleagues, made similar experiments harvesting Prague RSV particles at only 3 min intervals. They then compared the sedimentation properties and electrophoretic mobilities of the RNA in 3 min harvest virus with the RNAs of virus harvested at hourly intervals. They found the rapidly harvested virus contains a little 60-70S RNA but a large amount of $30-4 \mathrm{~S}$ RNA and a variable amount of 4-12S RNA. Furthermore, incubation at $40^{\circ} \mathrm{C}$ for $3 \mathrm{~min}$ of the $3 \mathrm{~min}$ harvest virus results in the conversion of most of the 30-40S RNA into 60-70S RNA.

These results suggest that $30-4 \mathrm{~S}$ RNA is a precursor to 60-70S RNA and, as Canaani et al. point out, the conversion may involve the association of 4S RNA. They say this for two reasons; first, when $60-70 S$ RNA is denatured back to $30-40$ S RNA five times more 4S RNA is released than is released when 30-40S RNA from $3 \mathrm{~min}$ virus is denatured directly; second, $30-40$ S RNA from 3 min virus is about five-fold less efficient as a template for reverse transcriptase than 60-70S RNA, and $4 \mathrm{~S}$ RNA is believed to act as a primer for reverse transcription. Of course, the report (Jarrett et al., 1971) that some free 30-40S RNA can be isolated directly from feline leukaemia virus particles harvested at long intervals gains significance in the light of these experiments.

\section{CEPHALOPODA \\ Deep-sea Cirromorphs}

DEEP-SEA cirromorphs (Cephalopda) have been recorded and photographed for the first time in the Arctic Ocean (Pearcy and Beal, Deep-sea Res., 20, $107 ; 1973)$. Out of more than 2,900
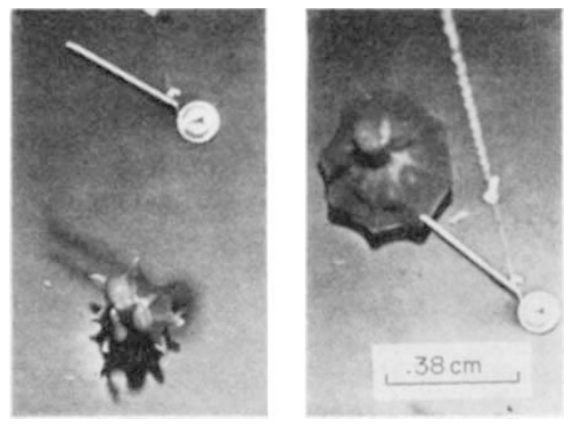

photographs taken off Point Barrow, Alaska, from USS Staten Island, cirromorphs appeared in twenty-one, representing a maximum of twelve individuals; all were at depths ranging from 3,219 to $3,786 \mathrm{~m}$.

The cirromorph in these photographs (taken $15 \mathrm{~s}$ apart) is swimming upright or perpendicular to the bottom, with its fins appearing to stroke horizontally. The arms and web in this sequence are first joined together and extend toward the bottom (left) and then expand laterally to form a large umbrella-like surface (right). A second mode of locomotion-swimming in the horizontal position, which is more typical of cephalopods-was also noted.

\title{
Initiation of Eukaryotic Protein Synthesis
}

NexT week, in Nature New Biology (March 14), Schreier and Staehelin present a detailed study of the roles of two purified protein factors involved in polypeptide chain initiation in eukaryotes. These authors suggest that the mRNA-independent binding of the initiator Met-RNAf to the 40S subunit is the first step in polypeptide chain initiation in eukaryotes and that this complex then directs the building of mRNA.

Four initiation factors, $\operatorname{IFE}_{1,2,3,4}$, were purified from rabbit reticulocytes by means of DEAE-cellulose followed by preparative glycerol density gradients. IFE, IFE 3 and GTP are absolutely required for the binding of Met-RNAf to $40 \mathrm{~S}$ subunits in the absence of mRNA. Two forms of IFE with sedimentation coefficients of $15 \mathrm{~S}$ and $17 \mathrm{~S}$ were detected, the smaller form probably being a degradative product of the larger.

With initiator Met-RNAf purified $\mathrm{IFE}_{2}$ but not $\mathrm{IFE}_{3}$ formed a complex which was GTP-dependent but ribosome and template-independent. In the presence of artificial template poly (A,U,G) and GTP, IFE 3 alone did not promote formation of the [40S Met-tRNA $A_{f}$ ] initiation complex, whereas $\mathrm{IFE}_{2}$ did. When natural globin mRNA replaced the artificial template, IFE 2 no longer promoted binding of Met-tRNAt and 40S subunits, but on the addition of $\mathrm{IFE}_{3}$ to $\mathrm{IFE}_{2}$ the complex was formed. Thus
$\mathrm{IFE}_{3}$ is required for binding of natural mRNA. Complex formation in the presence of $\mathrm{IFE}_{2}$ and $\mathrm{IFE}_{3}$ was just as efficient when mRNA was omitted. Thus $\mathrm{IFE}_{3}$ promotes template-independent binding of Met-tRNAf (presumably complexed with $\mathrm{IFE}_{2}$ and GTP) to $40 S$ subunits.

The [Met-tRNAf 40S] complex sedimented very close to the original $40 \mathrm{~S}$ subunits. But in the presence of excess $\mathrm{IFE}_{3}$, subunits which had not bound Met-tRNAf sedimented at about $48 \mathrm{~S}$, a sedimentation rate compatible with a particle composed of a 40 S subunit complexed with IFE. Schreier and Staehelin suggest that $\mathrm{IFE}_{3}$ combines initially with a 40 S subunit to direct the binding of Met-tRNAf. Subsequently the bulk of IFE $_{3}$ must dissociate from the initiation complex but a component of it may remain attached, to direct binding of mRNA. If $\mathrm{IFE}_{3}$ were heterogeneous with regard to such a component, this might provide a mechanism for mRNA selection.

In conclusion, IFE $_{3}$ promotes the mRNA-independent binding of MettRNA $_{f}$ to the $40 \mathrm{~S}$ ribosome subunit in the presence of IFE2. In that the initiator tRNA is bound first to the ribosome and helps the binding and correct phasing of messenger rather than the converse, this model of polypeptide chain initiation in eukaryotes is fundamentally different from the bacterial mechanism. 